\title{
Aplicaciones del internet en la actividad forestal, con especial referencia a México
}

\author{
Miguel Caballero Deloya ${ }^{1}$
}

\begin{abstract}
RESUMEN
Se describen brevemente los antecedentes de la Red o Telaraña Mundial de Información (World Wide Web: www) y se tocan aspectos acerca de su utilidad y funcionamiento, incluyendo las herramientas de búsqueda más comunes (motores); así mismo, se mencionan algunas de sus limitaciones. Se presentan algunas de las más útiles aplicaciones para el sector forestal de México, incluyendo los sitios de consulta considerados más importantes para el profesional forestal, estudiante, académico o persona interesada en el tema forestal. Las direcciones electrónicas incluidas abarcan instituciones nacionales, organizaciones internacionales, revistas especializadas e instituciones educativas y de investigación.
\end{abstract}

PALABRAS CLAVE:

Red Mundial de Información, motores de búsqueda, sector forestal, México, direcciones electrónicas.

\begin{abstract}
The background to the World Wide Web is presented along with aspects of its usefulness and way of working, including the most common search engines; also, some of its limitations are mentioned. Some of the most useful applications for the Mexican Forestry Sector are presented, including some of the most important web sites for the forest professional, student, researcher and the general public interested in the subject. The electronic addresses presented include national institutions, international organizations specialized journals and higher education and research institutions.
\end{abstract}

KEY WORDS:

World Wide Web, search engines, forestry sector, México, web sites. 


\section{INTRODUCCIÓN}

A partir de la mitad del siglo pasado, la sociedad mundial ha vivido notables cambios, motivados por un número de inventos y nuevas tecnologías que han transformado de manera sustancial sus hábitos y sistemas de vida. Pero de todos ellos, el que seguramente recibe la calificación de ser el más notable, es el binomio "computadora personal-Internet". Este inconmensurable desarrollo tecnológico ha roto todo tipo de fronteras geográficas en la comunicación, para dar lugar a lo que se ha dado en llamar la "autopista de la información”. Gracias a este notable avance, el ser humano de hoy puede estarse comunicando, enviando y recibiendo información con cualquier persona sin importar su ubicación geográfica, desde la comodidad de una oficina o aún de su propio hogar. También puede buscar todo tipo de información y recibirla unos minutos después, sin necesidad de tener que concurrir a una biblioteca o centro de documentación especializado.

Otro punto que es importante destacar, es que el universo de opciones de intercambio de información que se ha creado entre computadoras a partir del Internet, ha permitido un número de aplicaciones de gran valor como son: el correo electrónico, acceso a grupos de noticias y foros de debate, conversaciones (chat) y otras más.

Lamentablemente, la tecnología descrita ha tenido - y sigue teniendo - un desarrollo tan rápido, que a la fecha un gran sector de la población mundial no ha tenido tiempo u oportunidad para conocerla y aprovecharla en toda su potencialidad. México en este sentido, no es la excepción.

El presente trabajo busca orientar hacia la búsqueda y captura de información técnica en el campo de las ciencias forestales, de acuerdo a un enfoque de interés profesional o de trabajo, con base en el empleo de una computadora dotada de Internet.

El propósito es que esta información permita el acceso y facilite el empleo de una herramienta que hoy en día ha adquirido importancia estratégica e invaluable utilidad. Sin embargo, es conveniente aclarar que las instituciones, ejemplos y casos enlistados en la presente integración son sólo unos pocos ya que se trata de un esfuerzo inicial que, indudablemente, puede ampliarse progresivamente hasta constituir en un futuro cercano, un auténtico directorio.

\section{OBJETIVO}

Brindar a las personas ligadas a la actividad forestal y muy especialmente a los profesionales forestales mexicanos, información de base para el manejo y utilización del Internet en el proceso de obtener información técnica o científica de utilidad en el desempeño de su quehacer profesional.

Para alcanzar el objetivo propuesto, la presente aportación se ha integrado de dos partes: La primera, pretende orientar a los lectores, particularmente a aquellos que tienen poca experiencia en el empleo de Internet, sobre las características, la utilidad y el potencial, pero también algunas limitantes que caracterizan a esta valiosa herramienta. Uno de los aspectos de mayor interés de esta sección es la relativa al empleo de motores de búsqueda, con el propósito de facilitar el poder captar una mayor y una mejor información en el proceso de exploración. La segunda parte se ha orientado a aportar información sobre un conjunto de páginas Web, tanto para consulta general, como para obtener información especializada en el campo de las ciencias forestales. Con este propósito se han incluido algunas instituciones y organizaciones, 
tanto nacionales como internacionales de especial relevancia en el campo forestal. Se considera que este breve, pero selecto abanico institucional aquí integrado permitirá a los interesados ampliar la cobertura de información de acuerdo a sus necesidades.

\section{EL INTERNET}

\section{¿Qué es el Internet?}

Es una interconexión de redes informáticas que permite a las computadoras conectadas, comunicarse directamente, es decir, cada computadora de la red puede conectarse a cualquier otra computadora de la red (Enciclopedia Microsoft Encarta, 2002). En el presente, se ha constituido en una red internacional de computadoras que vincula miles de redes individuales de agencias como son: militares, gubernamentales, educativas, industriales, financieras, etc. así como empresas comerciales (proveedores de servicios) de todos tamaños y que permite a las personas acceder a la red (Columbia Encyclopedia, 2001). Su rápido desarrollo está teniendo importantes efectos sociales, económicos y culturales, convirtiéndose de esta manera en uno de los medios más influyentes de la llamada Sociedad de la Información y en la Autopista de la Información por excelencia (Fernández Calvo, 2003).

Nació en 1969 en los Estados Unidos como parte de un estudio de factibilidad secreta a cargo del Departamento de Defensa de ese país. El objetivo fue ensayar métodos que permitirían la supervivencia de redes de computadoras sujetas a ataques militares, recurriendo a un proceso dinámico de re-enrutamiento de mensajes (Columbia Enciclopedia, loc.cit.). Se inició con el nombre de ARPA (Advanced Research Projects Agency), bajo la forma de una red informática que conectaba computadoras localizadas en sitios dispersos y que operaban bajo diferentes sistemas operativos, con la ventaja de que cada computadora se podía conectar a las demás. La interconexión fue posible gracias al desarrollo de los protocolos IP y $\mathrm{TCP}^{1}$, por el Ingeniero estadounidense Robert Kahn. Tal desarrollo trascendió fuera del Departamento de Defensa, para conectar una gran cantidad instituciones educativas y de investigación, bajo el nombre de ARPAnet (Advanced Research Projects Agency Networks, nombre que subsistió hasta el año de 1974 (Fernández Calvo, 2001). Posteriormente, a dicha red se le sumaron nodos de Europa y del resto del mundo, para formar la Telaraña Mundial (World Wide Web) (Enciclopedia Microsoft Encarta, Loc.cit.).

\section{¿En qué consiste?}

El Internet es una "red de redes", que tiene la virtud de enlazar una enorme cantidad de computadoras que comparten los protocolos TCP/IP (Transmisión Control Program/Internet Protocol). Cada computadora puede acceder a la red a través de cables telefónicos o señales de radio e intercambiar información con otra terminal conectada con la red. De la misma manera, en cualquier computadora se puede constituir una base de datos particular y mantenerla en forma permanente, identificada con el nombre de "sitio" (Web site). Éste se identifica por una dirección a la cual puede acceder cualquier otra computadora conectada en la red. El proceso de conectarse de un sitio a otro se conoce como "navegación".

1 TCP/IP (Protocolo de Control de Transmisión/Protocolo Internet) Sistema de protocolos, definidos en RFC 793, en los que se basa buena parte de Internet. El primero se encarga de dividir la información en paquetes en origen, para luego recomponerla en destino, mientras que el segundo se responsabiliza de dirigirla adecuadamente a través de la red. Fuente: Fernández Calvo, R. 2001. Glosario básico inglés-español para usuarios de Internet. Madrid, España. 
Para auxiliar en la navegación, existen mecanismos especializados, de los cuales se habla más adelante (motores de búsqueda) (Anónimo, 2003).

\section{¿Cuál es su utilidad?}

Se ha señalado que, "aunque la interacción informática todavía está en su infancia, ha cambiado espectacularmente el mundo en que vivimos, eliminando las barreras del tiempo y la distancia y permitiendo a la gente compartir información y trabajar en colaboración» (Enciclopedia Microsoft Encarta, 2002).

En el campo de las ciencias hoy día, la disponibilidad de Internet brinda acceso a prácticamente toda la gama de materiales bibliográficos necesarios en la elaboración de escritos técnicos y científicos, como son: (a) artículos, libros y una amplia variedad de documentos e información sobre una gran variedad de temas, disponible en línea; (b) diccionarios y enciclopedias; (c) directorios de instituciones gubernamentales, del sector privado, ONGs, etc. así como de funcionarios, docentes, investigadores, etc. También se puede consultar información estadística, económica, censos, etc. al igual que legal (leyes y normas jurídicas) y muchas más.

La condición especial que se requiere para obtener el beneficio potencial del Internet, es el conocimiento apropiado y la capacidad para utilizar eficientemente la herramienta. Esto como ocurre en la mayoría de las situaciones, se logra con trabajo, práctica y paciencia.

\section{¿Cómo funciona?}

Hace la función de vehículo de transporte de información almacenada en archivos o documentos de otras computa- doras (University of California, 2003). De hecho, es un conjunto de redes locales conectadas entre sí a través de una computadora especial para cada red, conocida como «gateway» (puerta). Las interconexiones entre gateways se efectúan a través de diversas vías de comunicación, entre las que figuran líneas telefónicas, fibras ópticas y enlaces por radio. La información que se debe enviar a una máquina remota se etiqueta con la dirección computarizada de dicha máquina.

Una vez direccionada, la información sale de su red de origen a través de "la puerta". De allí es enviada de puerta en puerta hasta que llega a la red local que contiene la máquina de destino (Enciclopedia Microsoft Encarta, loc.cit.).

\section{La red o telaraña Mundial (World Wide Web)}

A finales de 1989, el informático británico Timothy Berners-Lee dio nacimiento a la "World Wide Web» (WWW) para la Organización Europea para la Investigación Nuclear (CERN). El propósito fue desarrollar una red que permitiese el intercambio de información entre los investigadores que participaban en proyectos vinculados a dicha organización (Enciclopedia Microsoft Encarta, loc.cit.).

La World Wide Web se conoce en el presente simplemente como «la Web» y ha sido bautizada en español como «la Malla o Telaraña Mundial». Ha sido definida como «una colección de textos, de documentos multimedia, de archivos y de otros servicios en red, de distribución global, interconectados de tal manera que constituyen una inmensa biblioteca electrónica que puede ser recuperada con rapidez por medio de búsquedas intuitivas» (Columbia Encyclopedia, 2001. Traducción al castellano por el autor). 
La Web representa la aplicación de lo que se conoce como "tecnología hipertexto" y de una "interfase gráfica a Internet" para la recuperación de información contenida en documentos con formato especial, que puede residir en la misma computadora o ser distribuidos a través de muchas computadoras alrededor del mundo. El «hipertexto» ha sido definido como «la capacidad de tener páginas Web que contienen enlaces, los cuales son áreas en una página, botones o gráficas, sobre los que se puede oprimir el botón del mouse para incorporar otro documento a su computadora» (Universidad de California, 2003).

La Web se apoya en tres elementos fundamentales:

(a)El HTML (Hypertext Markup Language - Lenguaje de Marcado de Hipertexto). Comprende una serie de códigos de programación que definen tipo de letra, diseños, gráficas y enlaces (hiperenlaces) para otros documentos accesibles vía la Web.

(b) EI HTTP (HypertText Transfer Protocol - Protocolo de Transferencia de Hipertexto). Define una serie de normas para transmitir páginas Web a través del Internet.

(c)El URL (Universal Resource Locator Localizador Universal de Recursos). Nombre estandarizado para identificar un documento o archivo en la Web. De hecho constituye la dirección de un enlace (Columbia Encyclopedia. loc.cit.). Cuando se oprime un enlace de cualquier tipo en una página Web, se envía una solicitud para capturar un documento único en alguna computadora específica en el mundo, que está identificado de manera exclusiva con ese URL (University of California, 2003). En un URL se especifican: (a) el protocolo de transferencia; (b) la dirección de Internet de la máquina que corresponde a la empresa, organización, persona, etc. de que se trata y (c) el nombre del fichero o archivo. Para ejemplificar, el URL a continuación: http:// www.conafor.gob.mx/documentos conafor/intro_pnf.htm especifica inicialmente el protocolo de transferencia (http); a continuación conduce a la dirección de la CONAFOR y dentro de su sistema, lleva finalmente al fichero mediante el cual se puede ingresar al Programa Nacional Forestal 20012006.

Los programas informáticos conocidos con el nombre de "exploradores», como es el caso de Netscape, Navigator o Internet Explorer (de Microsoft), recurren al protocolo http para recuperar los ficheros buscados.

\section{Motores o Sistemas de Búsqueda}

Cualquier búsqueda con el auxilio del Internet se basa en el empleo de «Sistemas o Motores de Búsqueda» (search engines). Un motor de búsqueda es "un programa de computación que se emplea para investigar una base de datos de documentos en Internet". De esta manera, la capacidad que un motor de búsqueda tiene para localizar la información de nuestro interés, depende de la naturaleza, el enfoque y la amplitud de la base de datos en la cual opera. Por este motivo, la mayor diferencia existente entre los motores de búsqueda radica en los criterios empleados para crear la base de datos.

La creación de bases de datos se apoya en dos procedimientos: a través del criterio humano o por medios automatizados. En el primer caso se habla de «Bases de Datos por Selección Humana» (Human-Selected Databases), en tanto que en el segundo, se hace referencia a «Bases de Datos Generados por Procedimientos Robóticos» (Robot-Generated Databases) (Cornell University, 2002). 
Lo señalado explica por qué los resultados de una búsqueda empleando diferentes motores, son siempre diferentes. También pone en evidencia tres aspectos importantes: (1) Ningún motor de búsqueda es perfecto; (b) Ninguna base de datos es completa y (c) Ninguna base de datos se encuentra actualizada.

En el presente, el número disponible de motores de búsqueda ha crecido notablemente y la información de sus capacidades y ventajas no es todavía conocida para la mayor parte de usuarios de la Web. Algunos de los motores más conocidos son: Google (google.com.mx); AltaVista (altavista.com); Yahoo (yahoo. com); Lycos (lycos.com); WebCrawler (webcrawler.com); Infoseek (infoseek.go. com); DejaNews (deafblind.com/deja2. html); Advanced Search (search.yahoo. com/search/options); Northern Light Power Search (northernlight.com/ power.html); Alltheweb (alltheweb.com/).

El proceso de desarrollo de los motores de búsqueda ofrece ahora algunos con enfoque geográfico (Yahoo China; Ananzi South Africa; Iran Index; MexicoGlobal; Lycos France; Excite Australia). Los hay también orientados a temas o disciplinas específicas (FirstGov; Search4Science; MedHunt; LawCrawler).

Recientemente ha surgido y crecido en importancia, una nueva categoría de buscadores bajo el nombre de «Motores de Búsqueda Meta» (Meta Search Engines), que tienen la particularidad más que de concentrarse en su propia base de datos, de apoyarse en otros motores de búsqueda para hurgar en diferentes bases de datos e integrar finalmente sus resultados. Estas herramientas son muy útiles para llevar a cabo investigaciones de temas o materiales que se sospecha existen en la Web. Están enfocadas a ahorrar tiempo haciendo posible la investigación por parte de una variedad de motores de búsqueda sin tener que conectarse secuencialmente a cada uno de ellos (Cornell University, 2002). Algunos ejemplos de motores de búsqueda meta son: Dogpile; Ixquick; Metacrawler; Profusion; Vivisimo.

\section{¿Cuáles son sus limitantes?}

Cazau (2003) ha resumido cuatro importante limitantes que presenta la navegación por Internet en la actualidad:

Dificultades en la búsqueda. No obstante todo el beneficio potencial que represente la búsqueda de información con el auxilio del Internet, este procedimiento puede consumir mucho tiempo y los resultados pueden ser pobres y poco satisfactorios. Parte del problema radica en el hecho de que, a diferencia de los procedimientos tradicionales para llevar a cabo consultas en la biblioteca, los criterios para buscar información navegando en Internet aún son muy recientes y a la fecha adolecen de muchas deficiencias. No obstante existen algunos elementos que auxilian en esta tarea para hacerla, dentro de las limitantes existentes, más productiva y eficiente. A los interesados en contar con una rápida y excelente guía didáctica (tutorial) sobre procedimientos y criterios para llevar a cabo una eficiente consulta en la $W e b$ se les sugiere consultar el trabajo de Chamberlain (2000).

Carencia de control de calidad en las publicaciones. A diferencia de las casas editoriales que elaboran libros o de las revistas periódicas de cierto prestigio que contienen comités editoriales, arbitraje, etc. que velan por la calidad de sus publicaciones, el material publicado por Internet no está sujeto a ningún tipo de control de calidad. En estas condiciones, el usuario debe asumir tal responsabilidad y actuar como su propio asesor de calidad (Cazau, 2003). 
Información de paga. El uso del Internet implica encontrar información tanto gratuita como de paga. En este caso, aunque en ocasiones los precios son razonables, no siempre ocurre así, resultando en frustración para el usuario del servicio, tanto por el costo del material requerido como por su desconocimiento de pago, particularmente cuando se trata de sitios internacionales. Normalmente, las revistas periódicas más prestigiadas están sujetas a un pago de suscripción en línea. En el caso forestal se pueden citar a manera de ejemplos, las revistas Journal of Forestry y Forest Science, ambas publicadas por la Sociedad de Forestales Americanos (Society of American Foresters). Se pueden obtener los resúmenes (Abstracts) de los artículos en línea gratuitamente, pero si se desea el artículo completo es necesario hacer el pago correspondiente.

Predominio del idioma inglés. Durante décadas, el inglés ha sido el idioma central en el cual se ha publicado la mayor parte de la literatura técnica y científica del mundo. Por el mismo motivo, la información y documentación que circula en el Internet se hace predominantemente también en el mismo idioma. Esto sin lugar a dudas, representa una gran limitante para los usuarios que carecen de dominio de dicho idioma.

\section{Aplicaciones}

Si bien el propósito del presente trabajo está orientado a brindar ejemplos prácticos y útiles sobre la aplicación del Internet en investigaciones y estudios forestales, este universo resulta tan amplio que sólo se han resumido algunos ejemplos relativos a diversas aplicaciones. El empleo de motores de búsqueda como ya se señaló puede resultar de gran utilidad para los interesados en inquirir sobre temas, institu- ciones y aspectos no cubiertos en esta sencilla presentación.

Consulta general. Se puede obtener información general de enciclopedias y diccionarios en línea, visitando el sitio: virtual.ues.edu.sv/ref/encydic/.

Diccionarios. Existen diversos tipos de diccionarios que funcionan "en línea" (a condición de estar conectado a la red). Algunos diccionarios del idioma español disponibles se pueden localizar en los sitios siguientes:

Diccionario de la Real Academia de la Lengua Española. Se pueden hacer consultas en línea en el sitio: encarta.msn.es/Default.asp.

Diccionarios Vox que Incluyen las siguientes modalidades: Español. Diccionario de la Lengua y de Sinónimos y Antónimos; InglésEspañol y Español-Inglés e InglésFrancés y Francés-Español. Sitio: diccionarios.com//index.phtml.

Enciclopedias. Se brinda a continuación un sitio que informa sobre enciclopedias y diccionarios en línea, la mayor parte en el idioma español: virtual.ues.edu.sv/r ef/encydic. Entre las fuentes de consulta más recomendables, se puede destacar a la Enciclopedia Encarta: encarta.msn.es/ Default.asp. En idioma inglés: Columbia Encyclopedia. 2002. Sexta Ed. New York. Contiene cerca de 51000 entradas y más de 80000 referencias cruzadas de hipertexto. Es una material de consulta de gran utilidad para quienes manejan el idioma inglés. Sitio: bastleby.com/65/.

\section{Búsqueda de información profesional}

Instituciones nacionales. Las instituciones nacionales a las que debe recurrir el técnico o el investigador forestal, según 
el tema de interés pueden ser muchas. A continuación se destacan sólo siete de ellas, en consideración de su estrecha relación con la actividad forestal. Se recomienda a los interesados en otras instituciones no incluidas en el presente resumen, recurrir a alguno de motores de búsqueda mencionados para localizar sus sitios Web correspondientes.

Banco de México. El Banco de México, una de las instituciones más importantes del país, tiene como cometido fundamental la procuración de la estabilidad del poder adquisitivo de la moneda nacional. Tiene a su cargo la rectoría de los sistemas financiero y monetario y las funciones regulatorias que en materia monetaria le son propias. Por lo anterior, visitar la página Web del Banco de México es de gran valor para el empresario, el industrial y el investigador que trabajan alrededor de los aspectos económicos de la actividad forestal. El Banco de México maneja y brinda información de los indicadores económicos y financieros del país. Entre ellos se pueden mencionar índices de precios, balanza de pagos, Producto Interno Bruto, volumen de la producción industrial, producción manufacturera, salarios, etc., independientemente de diferentes tipos de reportes que analizan el comportamiento de la economía nacional. Se puede visitar en el sitio: banxico.org. $m x /$.

\section{Comisión Nacional Forestal (CONAFOR). La Comisión Nacional} Forestal (CONAFOR), creada por Decreto Presidencial el 4 de abril del 2001, es un Organismo Público Descentralizado que ha venido instrumentando la política y la administración forestal del país. Información sobre los programas de desarrollo orientados a promover actividades productivas, así como de conservación forestal, además de las políticas nacionales en materia de desarrollo forestal sustentable a cargo de esta orga- nización, se pueden obtener en el sitio siguiente: conafor.gob.mex/.

Consejo Nacional de Ciencia y Tecnología (CONACYT). El Consejo Nacional de Ciencia y Tecnología es el instrumento del Gobierno Federal responsable de impulsar el desarrollo científico y tecnológico del país. Para ese propósito apoya estudios de posgrado, brinda estímulos económicos a las instituciones educativas y de investigación, financia proyectos de investigación y otras actividades semejantes. El sitio es: main. conacyt.mx.

\section{Instituto Nacional de Estadística, Geografía e Informática (INEGI). EI} Instituto Nacional de Estadística, Geografía e Informática tiene a su cargo los Sistemas Nacionales Estadístico y de Información Geográfica de México. Además promueve y orienta el desarrollo informático del país. Cuenta con un sistema de información estadística que aporta cifras de carácter demográfico, social y económico. Dispone también, de un sistema de información geográfico que brinda características geográficas y físicas del territorio nacional, así como sobre su flora y fauna. Ofrece diversos servicios informáticos en línea. Su sitio es: inegi.gob.mx.

Secretaría de Economía. La Secretaría de Economía es la dependencia del Poder Ejecutivo Federal que promueve la competitividad y el crecimiento económico de las empresas. Su misión es «crear las condiciones necesarias para fortalecer la competitividad, tanto en el mercado nacional e internacional, de todas las empresas del país; en particular de las micro, pequeñas y medianas. Instrumentar una nueva política de desarrollo empresarial que promueva la creación y consolidación de proyectos productivos que contribuyan al crecimiento económico sostenido y generen 
un mayor bienestar para todos los mexicanos". Indudablemente que el sitio Web de la Secretaría de Economía, tiene información de utilidad para el componente industrial y empresarial de la actividad forestal nacional: economia.gob.mx.

\section{Secretaría de Educación Pública} (SEP). La Secretaría de Educación Pública, como órgano rector y promotor de la educación en México, tiene una importante vinculación con el sector forestal a través de la Dirección General de Educación Tecnológica Agropecuaria (DGETA), dependencia responsable de la administración de varios centros educativos distribuidos en toda la geografía del país que imparten educación forestal en los niveles medio superior, licenciatura y posgrado. El sitio de la SEP es: sep.gob.mx. El sitio de la DGETA a su vez, se puede visitar en: sep.gob. $m x /$ wb2/sep/sep_386_dgeta.

\section{Secretaría de Medio Ambiente y} Recursos Naturales (SEMARNAT). La Secretaría de Medio Ambiente y de Recursos Naturales es la dependencia del Gobierno Mexicano responsable de llevar a la práctica la política nacional en materia de protección ambiental y de los recursos naturales. Su sitio es: semarnat. gob.mx. La SEMARNAT maneja el Sistema Nacional de Información Ambiental y Recursos Naturales (SNIARN), donde entre otras cosas, se aportan cifras y estadísticas relativas a los recursos forestales del país, proyectos y programas alrededor de los mismos, y sobre el sector forestal nacional (incluyendo estadísticas de producción e información económica). Se puede consultar visitando el sitio: semarnat.gob. $m x /$ sniarn/recursos_forestales.shtml.

\section{Organizaciones internacionales}

Se mencionan a continuación, 15 instituciones $u$ organismos internacio- nales que como resultado de diversas consultas, fueron señaladas como aquellas con las que los profesionales y los interesados en asuntos forestales de nuestro país, han tenido o hacia las cuales han evidenciado mayor interés.

Asociación Técnica Internacional de Bosques Tropicales (ATIBT). La Asociación Técnica Internacional de Bosques Tropicales, conocida por sus siglas en el idioma francés, ATIBT (Association Technique Internationale des Bois Tropicaux) es una ONG constituida por más de 200 miembros pertenecientes a cerca de 33 países productores y consumidores de madera tropical. Se creó en 1951 por demanda de la FAO y de la OCDE (Organización de Cooperación del Desarrollo Económico). Sus objetivos son: (a) Promover el manejo forestal sustentable; (b) Promover a las maderas tropicales como un recurso natural renovable; (c) Informar a sus miembros de los desarrollos importantes dentro de la industria forestal tropical; (d) Llevar la voz de la industria en reuniones, foros, etc. nacionales e internacionales $y$, (e) Desarrollar estándares profesionales e internacionales que faciliten el intercambio y el consumo de productos de maderas tropicales. La ATIBT tiene su sede en la ciudad de París, Francia. Su sitio es: perso.wanadoo.fr/atibt/ indexeng.htm.

Centro Agronómico Tropical de Investigación y Enseñanza (CATIE). El Centro Agronómico Tropical de Investigación y Enseñanza (CATIE), localizado en Turrialba, Costa Rica, es una institución internacional regional dedicada a la enseñanza de posgrado y a la investigación relacionada con los recursos tropicales de la Región Centroamericana y del Caribe. Es una de las organizaciones educativas y de investigación líderes en el campo tropical, con muchos años de estar formando especialistas para América Latina. Un número de 
Maestros en Ciencias de México se ha formado en la misma. Se puede localizar en el sitio: catie.ac.cr.

\section{Centro de Agroforestería Mundial} (World Agroforestry Center). El Centro de Agroforestería Mundial es el nombre que recientemente adoptó el que por años fue conocido como el Centro Internacional para Investigación en Agroforestería (ICRAF). Este se creó en 1978 por iniciativa del Centro de Investigación para el Desarrollo Internacional (IDRC) de Canadá para promover la investigación agroforestal en países en proceso de desarrollo. El Centro de Agroforestería Mundial es considerado como la institución líder a nivel mundial en investigación agroforestal para el desarrollo. Al igual que el CIFOR, forma parte del Grupo Consultor sobre Investigación Agrícola Internacional (CGIAR). Su sede se localiza en Nairobi, Kenya. Su sitio es: worldagroforestrycentre.org/.

Centro Forestal Mundial (WFC). El Centro Forestal Mundial, WFC (World Forestry Center) es una institución educativa no lucrativa, que se fundó en la ciudad de Portland, Oregon, Estados Unidos en el año de 1966. Consta de tres programas: (a) el Instituto Forestal Mundial; (b) Bosques demostrativos y (c) el Museo Centro para el Descubrimiento Forestal. Los directores actuales del Centro son de Autralia, Canadá, Chile, Finlandia, Alemania, Indonesia, Irlanda, Japón, Nueva Zelanda, Noruega, Malasia, México, Portugal, Rusia, Corea del Sur y los Estados Unidos. Su sitio Web es: worldforestry.org/.

\section{Centro para la Investigación Forestal Internacional (CIFOR). El Centro para la Investigación Forestal Internacional se localiza en Bogor, Indonesia y cuenta con oficinas regionales en Brasil, Camerún y Zimbabwe. Es una institución de investi- gación internacional y de conocimiento global comprometida con la conservación}

de los bosques y con el mejoramiento de la gente que habita los trópicos. Nació en 1993 por iniciativa del CGIAR (Consultative Group on International Agricultural Research), organización que agrupa a un número de instituciones de carácter internacional orientadas a la búsqueda de soluciones a la pobreza y al hambre mundial a través de la investigación agrícola. En el sitio del CIFOR se puede obtener, además de información de los proyectos de investigación que lleva a cabo esta organización, otro tipo de información forestal de actualidad como son reuniones, congresos, cursos, etc. así como de libros y publicaciones de interés sobre el tema. El sitio es: cifor.cgiar.org/.

Fondo Mundial para la Conservación de la Naturaleza (WWF). El Fondo Mundial para la Conservación de la Naturaleza (World Wildlife Fund, WWF) es una organización mundial que trabaja a nivel local a través de una red de oficinas de distribución internacional. Su misión es detener la degradación del ambiente natural del planeta y construir un futuro en el que los humanos vivan en armonía con la naturaleza mediante: la conservación de la diversidad biológica del mundo asegurando que el uso de los recursos naturales renovables sea sostenible promoviendo la reducción de la contaminación y del consumo inadecuado El WWW concentra su esfuerzo en seis programas: (a) Cambio Climático; (b) Bosques para la Vida; (c) Aguas con Vida; (d) Mares Amenazados; (e) Especies; (f) Tóxicos. El sitio internacional es: worldwildlife.org/. Existe un sitio para México: wwf.org.mx/index1.php.

Instituto Europeo de Investigación Forestal (EFI). Se localiza en Joensu, Finlandia. Es una organización no gubernamental independiente que lleva a cabo investigación forestal en Europa. Está constituida por un número de miembros que forman una extensa red de investigación en Europa y en otras naciones. El 
EFI desarrolla proyectos de investigación, compila información forestal y organiza reuniones en Europa. El objetivo del Instituto es llevar a cabo trabajos de investigación sobre: (a) política forestal, incluida su vertiente medioambiental; (b) ecología forestal, la multifuncionalidad de los bosques, los recursos y el estado de salud de los bosques europeos; (c) la oferta y la demanda de madera y otros productos forestales y (d) establecer previsiones sobre la evolución de los recursos forestales en Europa, así como su utilización. Más información del ERI se puede obtener en su sitio: efi.fi/.

Instituto Interamericano de Cooperación para la Agricultura (IICA). El IICA es el organismo especializado en la agricultura y el bienestar rural del Sistema Interamericano (IICA, 2003). Se fundó el 7 de octubre de 1942 en la ciudad de Turrialba, Costa Rica. Actualmente la sede se localiza en la ciudad de San José, en el mismo país. México es un país miembro y cuenta con su oficina nacional del IICA en la ciudad capital. La misión del IICA es: «Apoyar a los Estados Miembros en su búsqueda del progreso y de la prosperidad en el Hemisferio, por medio de la modernización del sector rural, de la promoción de la seguridad alimentaria y del desarrollo de un sector agropecuario competitivo, tecnológicamente preparado, ambientalmente administrado y socialmente equitativo para los pueblos de las Américas». Por lo anterior, aunque el mayor esfuerzo del IICA se desarrolla en el campo agropecuario, una de las áreas estratégicas en la que concentra su cooperación es en el Desarrollo Sostenible. Para los estudiosos de las ciencias forestales alrededor de ese tema, en el Instituto de referencia, se puede conseguir información y publicaciones de valor. Otra área estratégica del IICA, que puede interesar a los estudiosos del tema forestal es lo relativo a Educación y Capacitación. El sitio del
Instituto es: iica.int./. Para los interesados en contactar el sitio de la representación del IICA en México puede hacerlo visitando: iica.org. $m x$.

Instituto Internacional de Silvicultura Tropical. IITF. El Instituto Internacional de Silvicultura Tropical (IITF) es parte del Servicio Forestal, Departamento de Agricultura de los Estados Unidos. Se creó en el año de 1939. Se localiza en Río Piedras, Puerto Rico, sobre terrenos de la Estación Experimental Agrícola de la Universidad de Puerto Rico. El Instituto se dedica a realizar investigación y transferencia de tecnología en el campo de la Silvicultura tropical en un nivel internacional. Cuenta con más de sesenta años de experiencia aplicando investigación interdisciplinaria alrededor de los aspectos físicos, sociales y económicos del manejo forestal tropical. Lleva a cabo investigación cooperativa con más de 50 instituciones de diversos países. A nivel internacional, ha llevado a cabo consultas e investigación en las Islas del Caribe, México, Centroamérica, Sudamérica y algunas partes tropicales de Asia. Su sitio Web es: fs.fed.us/global/iitf/.

\section{Organización de las Naciones Unidas} para la Agricultura y la Alimentación (FAO). Se fundó en 1945, bajo el mandato de elevar los niveles de nutrición y de vida, así como de mejorar la productividad agrícola y las condiciones de las poblaciones rurales. En la actualidad es, dentro de las agencias especializadas de las Naciones Unidas, una de las más grandes y la que está especializada en agricultura, dasonomía, pesca y desarrollo rural. El sitio de la FAO es: fao.org/. Para los profesionales forestales de nuestro país, seguramente resultará de gran interés visitar el sitio forestal de esta organización donde se puede obtener información relevante de cada país, de reuniones y congresos forestales de importancia mundial, se puede visitar 
la revista forestal internacional de la organización en materia forestal, Unasylva, e información temática de interés actual (certificación forestal, biodiversidad, biotecnología, plantaciones forestales, investigación y educación, etc.). El sitio de referencia es: fao.org/forestry/ index.jsp.

\section{Organización Internacional de Maderas} Tropicales (ITTO). La Organización Internacional de Maderas Tropicales, más conocida por su denominación en idioma inglés como: International Tropical Timber Organization (ITTO), fue creada por el Acuerdo Internacional de Maderas Tropicales (ITTA) en 1983 bajo los auspicios de la Conferencia de las Naciones Unidas sobre Comercio y Desarrollo (UNCTAD) y empezó a operar en 1885. $\mathrm{Su}$ sede se estableció en Yokohama, Japón, en 1986. La idea fundamental que sirvió de base para la creación de esta organización, fue la de proporcionar un marco efectivo de consulta entre sus países miembros - productores y consumidores de maderas y productos maderables tropicales - en todos los aspectos relacionados con la economía mundial de dichos bienes. La misión de la agrupación es "facilitar la discusión, la consulta y la cooperación internacional de todos aquellos asuntos relacionados con el comercio internacional y la utilización de maderas tropicales, así como el manejo sustentable de su recurso base". El sitio Web de esta organización proporciona abundante información sobre ITTO y tópicos relacionados con el manejo de bosques tropicales en diferentes partes del mundo, al igual que en relación con el comercio mundial de productos forestales derivados de maderas tropicales: itto.or.jp/ index.html.

\section{Programa de las Naciones Unidas para} el Medio Ambiente. PNUMA. El Programa de las Naciones Unidas para el Medio Ambiente tiene como misión, el proporcionar liderazgo y promover la asociación para el cuidado del ambiente, inspirando, informando y capacitando a las naciones y a las gentes a mejorar su calidad de vida sin comprometer la de las generaciones futuras. Se puede obtener importante información de los programas que esta organización lleva a cabo a nivel mundial en cooperación con los gobiernos nacionales para el cuidado, protección y restauración de ecosistemas, biodiversidad, regiones naturales, comunidades bióticas, etc., en su sitio: unep.org/. También se resume información de leyes, convenciones internacionales y acciones que se llevan a cabo a nivel mundial con el mismo propósito.

Recursos para el Futuro (RFF). Recursos para el Futuro, cuyo nombre en el idioma inglés es Resources for the Future, es una organización apolítica, no lucrativa, que se fundó en el año de 1952, ubicada en la ciudad de Washington, D.C. en los Estados Unidos. Lleva a cabo investigación independiente en los temas de economía y ciencias sociales orientadas a asuntos estratégicos de interés ambiental y de los recursos naturales. La organización goza de prestigio por la calidad de sus investigaciones, gran parte de ellas, relacionados con bosques y recursos naturales. Además cuenta con buena cantidad de información documental y publicaciones que se pueden consultar vía Internet. Su sitio es: rff.org/.

Servicio Forestal de los Estados Unidos (US Forest Service). Por su antigüedad, desarrollo, organización y nivel tecnológico, visitar el sitio Web del Servicio Forestal de los Estados Unidos es de gran utilidad para los investigadores, académicos y profesionales, y en general todo tipo de personas ligadas a la actividad forestal. Información de especial utilidad es la relativa a políticas nacionales en materia forestal, información estadística, proyectos en marcha, programas de investigación y de manejo, etc. El sitio es: fs.fed.us/. Considerando que el sistema de investigación forestal 
de los Estados Unidos es el mayor del mundo en su género (presupuesto, infraestructura, número de investigadores, cobertura temática, etc.), para los investigadores, académicos y estudiantes, visitar el sitio del servicio forestal estadounidense de este interesante tema, seguramente que sería valioso y constituiría un punto de partida para obtener información específica sobre temas tecnológicos y científicos de interés particular: fs.fed.us/research/scientific.html.

\section{Unión Internacional de Organizaciones} de Investigación Forestal (IUFRO). La Unión Internacional de Organizaciones de Investigación Forestal (IUFRO) es, para todo investigador en ciencias forestales, una organización especialmente importante y estratégica. Se define a sí misma como: «Una red internacional no lucrativa, no gubernamental de científicos forestales». Tiene su sede en Viena, Austria. Sus objetivos son promover la cooperación internacional en investigación en la Dasonomía y productos forestales. Se creó en 1892 y tiene una presencia importante en todo el mundo. Integra a más de 15000 científicos en calidad de miembros y más de 700 instituciones en más de 100 naciones. Visitar el sitio Web de la IUFRO puede aportar información, ideas, contactos, etc. a los interesados en las ciencias forestales (IUFRO, 1996). Su sitio en la red es: iufro. boku.ac.at/iufro/secre/introduction.htm.

\section{Revistas especializadas}

En la actualidad existe una gran cantidad de revistas especializadas en diversas ramas de la ciencia forestal. La presente revisión sólo hace mención de seis de ellas. Los interesados en tener información del universo de revistas que se publican en el orbe en el tema de referencia pueden consultar alguno de los dos sitios que se indican a continuación. El primero es la Biblioteca virtual de la
Web que se elabora en Finlandia y da conocer una lista de publicaciones periódicas exclusivamente en el campo de las ciencias forestales: metla.fi/info/vlib/ Forestry/Category/Publications/Journals. La segunda fuente se conoce como Medbioworld, la que divulga una relación combinada de revistas forestales y de horticultura en el siguiente sitio Web: medbioworld.com/bio/journals/ biojournals.html.

Índice de Revistas Mexicanas de Investigación Científica y Tecnológica que da a conocer el CONACYT (última actualización al 6 de marzo de 2003), lamentablemente no incluye más que una revista nacional que aborde la temática forestal y que satisfaga los niveles de calidad establecidos por el Consejo (conacyt.mx/daic/. Se trata de la revista Agrociencia que se reseña a continuación. Por este motivo, el resto de revistas que se incluyen en este apartado, son todas de carácter internacional.

Agrociencia. Revista científica creada y respaldada por el Colegio de Postgraduados con la finalidad de difundir los resultados de la investigación agropecuaria y forestal, tanto de investigadores mexicanos como de otros países. Toda contribución es sometida a un estricto arbitraje. A partir del año 2000 su periodicidad es bimestral y en formato totalmente bilingüe (español e inglés en el mismo ejemplar). Además de la versión impresa, el contenido completo está disponible en su versión electrónica, sin costo adicional. El sitio es: colpos. $m x /$ agrocien/Bimestral/Contenido.html.

Agroforestería en las Américas. Revista periódica en español que publica el CATIE. Tiene periodicidad trimestral. Está orientada a divulgar los avances y aplicaciones de la agroforestería en los países del continente americano. Su propósito es promover un aprovechamiento de los recursos naturales más 
acorde a las posibilidades ecológicas y a las necesidades sociales de los países de la región, así como favorecer el intercambio de conocimientos y una mayor cooperación entre las instituciones y grupos involucrados con la actividad agroforestal. El sito Web es: catie.ac.cr/ información/RAFA/Principal.htm.

Forest Science. Al igual que la revista Journal of Forestry, Forest Science es un órgano de la Sociedad de Forestales Americanos de Estados Unidos (Society of American Foresters). Es una de las publicaciones periódicas (bimestral) con liderazgo internacional en materia de investigación forestal. Lleva más de 45 años publicando artículos científicos con enfoque en las áreas de silvicultura, suelos forestales, biometría, sanidad forestal, recreación en ambientes naturales, fisiología de árboles, manejo de bosques, extracción y transporte de productos y análisis de políticas forestales. El domicilio de suscripción es el mismo (dirección oficial de la SAF) anotado para la revista Journal of Forestry.

Forestry. Esta revista periódica del Reino Unido es publicada por la universidad de Oxford (Oxford University Press). Divulga artículos con arbitraje sobre investigación, con relación a la práctica y políticas que promuevan el desarrollo sustentable de los bosques, los terrenos forestales y los árboles. Para la selección de artículos por publicar, le dan prioridad a la originalidad de las contribuciones y la aplicación práctica. Aunque dan preferencia a trabajos desarrollados en las zonas templadas y boreales, también aceptan artículos que reúnan méritos, relativos a zonas tropicales. Visitando el sitio Web, es posible consultar resúmenes y frecuentemente versiones completas de los artículos publicados en cada número. Al momento de elaborar el presente manuscrito está disponible el primer número (Vol 76. No. 1) del 2003. El sitio de referencia es: www3.oup.co.uk/foresj/.

Journal of Forestry. Órgano oficial de la Sociedad de Forestales Americanos, Estados Unidos. Publicación de larga tradición y antigüedad. Su misión es promover el avance de la profesión forestal a través de la información a los profesionales forestales sobre desarrollos significativos e ideas en la ciencia forestal, en el manejo de los recursos naturales y política forestal. Responsable de la publicación: Society of American Foresters (Sociedad de Forestales Americanos). Su sitio en la red es safnet.org/pubs/periodicals.html.

Revista Forestal Centroamericana. Es una revista de carácter trimestral, editada en el idioma español que publica el Centro Agronómico Tropical de Investigación y Enseñanza (CATIE). Aunque su cobertura es fundamentalmente centroamericana, con frecuencia divulga información de otras regiones. Su enfoque primordial es hacia la conservación, el manejo y el aprovechamiento de los recursos naturales, temas ambientales y desarrollo rural, con especial énfasis a la región tropical. Se puede localizar en el sitio catie.ac.cr/informacion/RFCA/.

Unasylva. Unasylva es la revista periódica de la FAO especializada en la temática forestal (bosques e industrias forestales). Se publica trimestralmente en los idiomas inglés, español y francés. Normalmente, cada número está dedicado al análisis de un tema de interés estratégico en particular; así, el cuarto (último) número correspondiente al año 2002, se enfocó al tópico: "Energía de la Madera". Es posible leer e imprimir los artículos deseados visitando el sitio de la revista: fao.org/forestry/foris/webview/ forestry2/index.jsp? siteld=2342\&langld=1 . 


\section{Instituciones educativas}

La información que se presenta enseguida incluye fundamentalmente instituciones educativas nacionales que imparten educación de licenciatura y de posgrado en las ciencias forestales. Los interesados, a través de visitas a los sitios Web correspondientes, podrán obtener información de detalle de los enfoques y la naturaleza de los programas académicos.

Colegio de Postgraduados. Esta importante institución, además de ser pionera en ofertar el posgrado forestal en el país (a partir del año de 1979), es hoy la más destacada en este nivel educativo. Cuenta con el mayor número de estudiantes en el mismo, especializándose en cuatro áreas: (a) Silvicultura y Manejo de Bosques Naturales y Plantaciones; (b) Genética Forestal; (c) Ecología Forestal y (d) Economía Forestal. Su destacado desarrollo en educación de posgrado en los campos agrícola, pecuario, forestal y de los recursos naturales, la coloca como un centro académico de excelencia para el desarrollo rural nacional. Su sitio es: colpos. $m x /$. El sitio que corresponde concretamente al Programa Forestal es: colpos.mx/IRENAT/for/for_ind.htm.

\section{Universidad Autónoma Chapingo} (UACH). Del universo de instituciones educativas mexicanas en el campo de las ciencias forestales destaca la Universidad Autónoma Chapingo como la más relevante por diversos motivos. Fue la primera y por muchos años la única que en el contexto nacional, ofreció la licenciatura forestal. Actualmente es la que cuenta con mayores opciones en dicha carrera: (a) Ingeniero Forestal; (b) Ingeniero Forestal Industrial y (c) Ingeniero en Restauración Forestal. Por otro lado es la institución educativa que cuenta con el mayor número de alumnos, de profesores y de infraestructura, además de un excelente posgrado en materia forestal. Su sitio es: chapingo. $m x /$. Para quién desee contactar directamente a la División de Ciencias Forestales de esta misma institución, puede visitar el sitio: chapingo. $\mathrm{mx} /$ dicifo/.

Otras Universidades Nacionales. Un estudio realizado por el autor para la Comisión Forestal Nacional (Caballero, 2003), reveló que existen en el presente, 16 programas de licenciatura forestal que ofertan 14 planteles correspondientes a diez instituciones educativas. Por cuanto se refiere al posgrado en ciencias forestales, existen diez instituciones educativas que cuentan con programas académicos de maestría. Algunas de las instituciones más relevantes por cuanto se refiere a sus programas académicos, profesorado y solidez en su infraestructura para la enseñanza, se resumen en la Tabla 1, en el cual se incluye también el sitio Web institucional.

\section{Universidades del Extranjero}

En el contexto internacional, existe un grupo de universidades que sobresalen entre las más relevantes por su destacada aportación en el campo de las ciencias forestales, tanto desde el punto de vista académico como de la investigación. En ellas se ha formado un buen número de especialistas mexicanos en la materia. Dada la dificultad de enumerarlas a todas, solamente se incluyen algunas, acompañadas de su sitio Web en las Tablas 2 y 3 , universidades estadounidenses y universidades europeas respectivamente. Los interesados en contar con información detallada de las instituciones estadounidenses más relevantes que ofertan educación forestal de licenciatura y posgrado, pueden visitar el sitio de la Asociación Nacional de Escuelas y Facultades Profesionales Forestales (National Association of Professional Forestry Schools and Colleges) de ese país: napfsc.org/. 
Tabla 1. Algunas instituciones de Educación Superior que ofrecen Programas de Licenciatura y de Posgrado en Ciencias Forestales en México ${ }^{1}$

\begin{tabular}{|c|c|}
\hline INSTITUCIÓN & SITIO WEB \\
\hline $\begin{array}{l}\text { Colegio de Postgraduados** } \\
\text { Programa Forestal }\end{array}$ & colpos. $m x /$ \\
\hline $\begin{array}{l}\text { Secretaría de Educación Pública. } \\
\text { Dirección General de Educación Tecnológica } \\
\text { Agropecuaria (DGETA)* }\end{array}$ & $\begin{array}{l}\text { sep.gob.mx } \\
\text { sep.gob.mx/wb2/sep/sep_386_dgeta }\end{array}$ \\
\hline $\begin{array}{l}\text { Universidad Autónoma Agraria Antonio Narro. } \\
\text { UAAAN*. Departamento Forestal Saltillo, Coah. }\end{array}$ & uaaan.mx/index_01.htm \\
\hline $\begin{array}{l}\text { Universidad Autónoma Chapingo (UACH) } \\
\text { División de Ciencias Forestales (DICIFO) }\end{array}$ & $\begin{array}{l}\text { chapingo. } m x / \\
\text { chapingo. } m x / \text { dicifo/ }\end{array}$ \\
\hline $\begin{array}{l}\text { Universidad Autónoma de Chihuahua*. Facultad } \\
\text { de Ciencias Agrícolas y Forestales. } \\
\text { Delicias, Chihuahua. }\end{array}$ & uachnet.mx/ \\
\hline $\begin{array}{l}\text { Universidad Autónoma de Nuevo León*. } \\
\text { Facultad de Ciencias Forestales. } \\
\text { Linares, Nuevo León. }\end{array}$ & uanl.mx/ \\
\hline $\begin{array}{l}\text { Universidad Autónoma del Estado de Hidalgo. } \\
\text { Pachuca, Hidalgo. }\end{array}$ & reduaeh.mx/ \\
\hline $\begin{array}{l}\text { Universidad Autónoma Indígena de México } \\
\text { (UAIM) }\end{array}$ & uaim.edu.mx/academico.htm \\
\hline $\begin{array}{l}\text { Universidad de Guadalajara*. Centro } \\
\text { Universitario de Ciencias Biológicas y } \\
\text { Agropecuarias (CUCBA).Guadalajara, Jal. }\end{array}$ & udg. $m x /$ \\
\hline $\begin{array}{l}\text { Universidad de Guadalajara } \\
\text { Departamento de Madera, Celulosa y Papel. } \\
\text { Centro Universitario de Ciencias Exactas e } \\
\text { Ingenierías (CUCEI)** }\end{array}$ & dmcyp.cucei.udg.mx/www/index.asp \\
\hline $\begin{array}{l}\text { Universidad del Mar. Campus } \\
\text { Puerto Escondido. Oaxaca. }\end{array}$ & umar.mx/ \\
\hline $\begin{array}{l}\text { Universidad Juárez del Estado de Durango. } \\
\text { Escuela de Ciencias Forestales. } \\
\text { Durango, Durango. }\end{array}$ & ujed.mx/ \\
\hline $\begin{array}{l}\text { Universidad Michoacana de San Nicolás de } \\
\text { Hidalgo*. Facultad de Ingeniería en Tecnología } \\
\text { de la Madera. } \\
\text { Morelia, Michoacán. }\end{array}$ & ccu.umich.mx/ \\
\hline Universidad Veracruzana** & uv. $m x$ \\
\hline \multicolumn{2}{|c|}{$\begin{array}{l}1 \text { Para estar acordes con el presente análisis, solamente se han incluido instituciones que cuentan con página Web } \\
\text { ** Instituciones que ofertan educación forestal en los niveles de licenciatura y de posgrado. } \\
\text { ** Solamente se ofrece posgrado forestal. } \\
\text { Nota: La Comisión Nacional Forestal en su sitio: conafor.gob.mx/programas_nacionales_forestales/imasd /IEAF.htm } \\
\text { resume sitios Web de un número de instituciones educativas de México y del extranjero, que ofrecen grados } \\
\text { académicos o cursos relevantes en ciencias forestales. }\end{array}$} \\
\hline
\end{tabular}




\section{Instituciones de Investigación}

En México no existen instituciones que se dediquen a hacer investigación forestal en exclusividad. Existió una en el pasado, el desaparecido Instituto Nacional de Investigaciones Forestales. Por este hecho, las organizaciones que se mencionan en este apartado, son aquellas que dentro de sus programas generales de investigación, contemplan diversos aspectos de investigación forestal. Bajo esta perspectiva, podrían citarse varias organizaciones más, particularmente del contexto educativo. Sin embargo, el limitado universo de instituciones que se mencionan a continuación, ha demostrado especial relevancia en su quehacer científico en algunas ramas o temas forestales.

Tabla 2. Algunas Universidades de importancia en los Estados Unidos en el campo de la Educación Forestal y de los Recursos Naturales

\begin{tabular}{ll}
\hline UNIVERSIDAD & SITIO WEB \\
\hline $\begin{array}{l}\text { Colorado State University. } \\
\text { Fort Collins. Colorado. }\end{array}$ & welcome.colostate.edu/ \\
\hline $\begin{array}{l}\text { Michigan State University. } \\
\text { East Lansing, Michigan. }\end{array}$ & msu.edu/home/ \\
\hline $\begin{array}{l}\text { North Carolina State University. } \\
\text { Raleigh, N.C. }\end{array}$ & ncsu.edu/ \\
\hline $\begin{array}{l}\text { Oregon State University. } \\
\text { Corvallis, Oregon. }\end{array}$ & cof.orst.edu/ \\
\hline $\begin{array}{l}\text { Purdue University. } \\
\text { West Lafayette, Indiana. }\end{array}$ & purdue.edu/ \\
\hline $\begin{array}{l}\text { Syracuse University. } \\
\text { Syracuse, Nueva York. }\end{array}$ & syr.edu/ \\
\hline $\begin{array}{l}\text { University of California. } \\
\text { Berkeley, California. }\end{array}$ & berkeley.edu/ \\
\hline $\begin{array}{l}\text { University of Florida. } \\
\text { Gainesville, Florida. }\end{array}$ & ufl.edu \\
\hline $\begin{array}{l}\text { University of Idaho. } \\
\text { Moscow, Idaho. }\end{array}$ & uidaho.edu/ \\
\hline $\begin{array}{l}\text { University of Michigan. } \\
\text { Ann Arbor, Michigan. }\end{array}$ & umich.edu/ \\
\hline $\begin{array}{l}\text { University of Minnesota. } \\
\text { St. Paul, Minnesota. }\end{array}$ & www.1.umn.edu/ \\
\hline $\begin{array}{l}\text { University of Washington. } \\
\text { Seattle, Washington. }\end{array}$ & \\
\hline \begin{tabular}{l} 
New Haven, University. \\
\hline
\end{tabular} & \\
\hline
\end{tabular}


Tabla 3. Algunas Universidades importantes en Europa en el campo de la Educación Forestal y de los Recursos Naturales

\begin{tabular}{|c|c|c|}
\hline PAÍS & UNIVERSIDAD & SITIO WEB \\
\hline \multirow[t]{2}{*}{ Alemania } & Göttingen & uni-goettingen.de/ \\
\hline & $\begin{array}{l}\text { Dresden University of } \\
\text { Technology }\end{array}$ & $\begin{array}{l}\text { tu-dresden.de/cgi- } \\
\text { bin/htimage/banner.conf?69,12 }\end{array}$ \\
\hline Bélgica & $\begin{array}{l}\text { Facultad Universitaria de } \\
\text { Ciencias Agronómicas de } \\
\text { Gembloux }\end{array}$ & fsagx.ac.be/gf/ \\
\hline \multirow[t]{2}{*}{ España } & $\begin{array}{l}\text { Escuela Técnica Superior de } \\
\text { Ingenieros de Montes }\end{array}$ & montes.upm.es/ \\
\hline & $\begin{array}{l}\text { Universidad Politécnica de } \\
\text { Valencia }\end{array}$ & $\begin{array}{l}\text { 1.universia.net/CatalogaXXI/C100 } \\
\text { 38PPESI/1/E47358/index.html }\end{array}$ \\
\hline \multirow[t]{2}{*}{ Finlandia } & Joensuu & joensuu.fi/englishindex.html \\
\hline & Universidad of Helsinki & helsinki.fi/university/ \\
\hline Francia & $\begin{array}{l}\text { Ecole Nationale du Génie Rural } \\
\text { des Eaux et des Forêts } \\
\text { (ENGREF) }\end{array}$ & engref.fr/ANGLAIS/missions.htm \\
\hline Países Bajos & Wageningen. & wau.nl/welcome.html \\
\hline \multirow[t]{2}{*}{ Reino Unido } & Oxford & ox.ac.uk/ \\
\hline & Univ. De Gales. Bangor. & safs.bangor.ac.uk/ \\
\hline Suecia & $\begin{array}{l}\text { Universidad Sueca de Ciencias } \\
\text { Agrícolas. Umea }\end{array}$ & -umea.slu.se/ \\
\hline
\end{tabular}

\section{Centro de Investigación Científica de} Yucatán (CICY). Este centro de investigación y de educación, localizado en Mérida, Yucatán, se creó en 1979 como respuesta a la necesidad de apoyar las investigaciones sobre el henequén, a la postre, un cultivo estratégico en la Península de Yucatán. Sin embargo, durante los últimos años redefinió sus objetivos para atender las nuevas demandas de la región y del país. En el presente, el CICY es una institución nacional de avanzada en temas como: Biología Molecular; Bioquímica; Biotecnología; Ecología; Genética y Fisiología de Plantas, así como en la Química Orgánica; en la Química de las Macromoléculas y en el Desarrollo de Procesos.
Colegio de la Frontera Sur (ECOSUR). El Colegio de la Frontera Sur es un centro de investigación y educación a nivel posgrado, enfocado en el desarrollo y vinculación de México en su frontera sur. Sus programas se orientan a la generación de conocimientos científicos la formación de recursos humanos y el diseño de tecnologías y estrategias para la innovación orientada al desarrollo sustentable. ECOSUR ofrece maestría en Ciencias en Recursos Naturales y Desarrollo Rural y el Doctorado en Ciencias en Ecología y Desarrollo Sustentable. Su sitio es: ecosur.mx

Instituto de Ecología, A.C. (INECOL). Es un centro estratégico para el 
desarrollo social, la conservación del patrimonio natural y la preparación de profesionales, sostenido por la producción de conocimiento ecológico y de la biodiversidad, con reconocimiento internacional. Sus objetivos son: (a) Producir conocimiento científico; (b) Preparar profesionales de alta calidad; (c) Fortalecer la vinculación con los sectores productivo, público y social y (d) Conservar las colecciones biológicas y el patrimonio natural a su cargo. EI INECOL ofrece Maestría en Ciencias y Doctorado en Ciencias en Ecología y Manejo de Recursos Naturales. Su sitio es: ecologia.edu.mx/.

\section{Instituto Nacional de Investigaciones} Forestales, Agrícolas y Pecuarias (INIFAP). Es la organización nacional responsable de hacer e impulsar la investigación y el desarrollo tecnológico del país en los campos agropecuario y forestal. Su misión es generar conocimientos e innovaciones tecnológicas y promover su transferencia, considerando un enfoque que integre, desde el productor primario hasta el consumidor final, para contribuir al desarrollo productivo, competitivo, equitativo y sustentable del sector forestal, agrícola y pecuario en beneficio de la sociedad. Sus objetivos contemplan: (a) Generar conocimientos e innovaciones tecnológicas; (b) Desarrollar y promover investigación estratégica y de frontera; (c) Promover y apoyar la transferencia de conocimientos $y$ tecnologías, y (d) Fortalecer la capacidad institucional. La unidad encargada de la investigación forestal en el instituto es la Dirección General Forestal. El sitio Web del INIFAP es: inifap.conacyt.mx.

\section{Otros Sitios de Interés}

Consejo Técnico Consultivo Nacional Forestal (CONAF). El Consejo Técnico Consultivo Nacional Forestal (CONAF) constituye un espacio nacional de concer- tación de las organizaciones, instituciones, y asociaciones vinculadas con el desarrollo sustentable de los recursos naturales. Este órgano colegiado propicia la instauración de procesos de corresponsabilidad social e institucional para definir políticas y programas consensuados que permitan el aprovechamiento sustentable de los recursos forestales de México. El Consejo se constituyó como auxiliar de la SEMARNAT para identificar áreas prioritarias en materia forestal en las que deberán apoyarse actividades de investigación y formación de recursos humanos, y asegurar que la emisión y actualización de normas corresponda a los últimos avances del conocimiento en la materia. El sitio Web de este Consejo es: semarnat.gob. $m x / p a r t i c i p a c i o n$ ciudadana/consejo_forestal.shtml.

Forest Stewardship Council (FSC). El FSC es una organización internacional no lucrativa, que se fundó en 1993 para apoyar un manejo de los bosques del mundo en forma ambientalmente apropiada, socialmente benéfica y económicamente viable. Es una asociación de miembros que integra representantes de diversos grupos ambientales y sociales, profesionales forestales, organizaciones de grupos étnicos, de comunidades forestales y organizaciones certificadoras de productos forestales de todo el mundo. El FSC cuenta con su oficina operativa en la ciudad de Oaxaca, México. Más información de esta organización se puede obtener visitando su sitio: fscoax.org/ principal.htm.

\section{Directorios Forestales}

En el quehacer laboral surge la necesidad de consultar directorios de diferente naturaleza. Pueden ser de instituciones académicas, de industrias, comerciales, de investigación o asociaciones de diversos tipos. El Internet ofrece una valiosa herramienta de 
consulta a través del Proyecto de Directorio Abierto (totalmente gratuito) dmoz Open Directory Project - y de amplia cobertura, donde se pueden consultar directorios sobre diversas actividades 0 giros forestales en el sitio: search.dmoz. org/cgi-bin/search? search=forestry.

Un directorio de especial utilidad, particularmente para los interesados en el área industrial y comercial, es el Directorio de Steve Shook sobre productos forestales, ciencia de la madera y mercadeo de dichos productos. Es una lista de sitios Web y de otros recursos de Internet que también incluye una lista de publicaciones y de sitios relacionados con la certificación de productos forestales. Se puede consultar en el sitio: forestdirectory.com/.

\section{REFERENCIAS}

Anónimo. 2003. Computadora.inventos. hypermart.net/computadora.htm Accesado el 19 de marzo de 2003.

Caballero Deloya, M. 2003. Diagnóstico de la Educación y la Capacitación Forestal en México. Comisión Nacional Forestal-Colegio de Postgraduados. Guadalajara, Jal. Inédito. 111p.

Cazau, P. 2003. Las Publicaciones Científicas en Internet. Guía de Redacción y Publicación Científica. galeon. hispavista.com/pcazau/guia_red.htm Accesado el 21 de enero, 2003.

Chamberlain, Ellen. 2000. Bare Bones 101: A Very Basic Web Search Tutorial. Beaufort Library. University of South Carolina. Estados Unidos. sc.edu/beaufort/library/bones.html Accesado el 4 de abril, 2003.

Cornell University Library. 2002. What is an Internet Search Engine? mannlib.cornell.edu/reference/tutorials/search/engines.html. Ithaca, Nueva York. EUA. Accesado el 7 de febrero, 2003.

Enciclopedia Microsoft Encarta. 2002. Internet. encarta.msn.es. 1997-2002 Microsoft Corporation. Accesado el 25 de marzo, 2003.

Fernández Calvo, Rafael. 2001. Glosario Básico Inglés-Español para Usuarios de Internet. ati.es/novatica/glosario internet.html. Madrid, España. Accesado el 4 de marzo, 2003.

International Union of Forestry Research Organizations. 1996. The Secretariat. iufro.boku.ac.at/iufro/secre/introduction.htm Actualizado el 20/02/03. Accesado el 26 de marzo, 2003.

Metla Library Information Systems. 2003. Virtual Library: Forestry. University Departments and Research Groups. fsagx.ac.be/gf/.» 